\title{
Knoevenagel condensation for modifying the reducing endgroups of cellulose nanocrystals
}

\section{Heise, Katje}

2019-12-02

Heise , K, Koso , T , Pitkänen , L , Potthast , A , King , A W T , Kostiainen , M A \& Kontturi , E 2019 , ' Knoevenagel condensation for modifying the reducing endgroups of cellulose nanocrystals ' , Acs macro letters , vol. 8 , no. 12 , pp. 1642-1647 . https://doi.org/10.1021/acsmacrolett.9b00838

http://hdl.handle.net/10138/309564

https://doi.org/10.1021/acsmacrolett.9b00838

acceptedVersion

Downloaded from Helda, University of Helsinki institutional repository.

This is an electronic reprint of the original article.

This reprint may differ from the original in pagination and typographic detail.

Please cite the original version. 
This document is confidential and is proprietary to the American Chemical Society and its authors. Do not copy or disclose without written permission. If you have received this item in error, notify the sender and delete all copies.

\section{Knoevenagel condensation for modifying the reducing end- groups of cellulose nanocrystals}

\begin{tabular}{|r|l|}
\hline Journal: & ACS Macro Letters \\
\hline Manuscript ID & mz-2019-00838m.R1 \\
\hline Manuscript Type: & Letter \\
\hline Author: & n/a \\
\hline & $\begin{array}{l}\text { Complete List of Authors: } \\
\text { Koso, Tetyana; University of Helsinki } \\
\text { Pothast, Leena; Aalto-yliopisto, Bioproducts and Bond Bepartment of Chemistry, Division of Chemistry } \\
\text { King, Alistair; Helsingin Yliopisto, Chemistry } \\
\text { Kostiainen, Mauri; Aalto University, Department of Biotechnology and } \\
\text { Chemical Technology } \\
\text { Kontturi, Eero; Aalto-yliopisto, Bioproducts and Biosystems }\end{array}$ \\
\hline
\end{tabular}

\section{SCHOLARONE" Manuscripts}




\title{
Knoevenagel condensation for modifying the reducing end-groups of cellulose nanocrystals
}

\author{
Katja Heise ${ }^{\dagger}$, Tetyana Koso ${ }^{\ddagger}$, Leena Pitkänen ${ }^{\dagger}$, Antje Potthast ${ }^{\tau}$, Alistair W. T. King ${ }^{\ddagger}$, Mauri A. \\ Kostiainen $^{\text {t** }^{*} \text { and Eero Kontturi }}{ }^{\dagger^{*}}$ \\ ${ }^{\dagger}$ Department of Bioproducts and Biosystems, Aalto University, P.O. Box 16300, FI-00076 Aalto, Espoo, Finland \\ ¥ Materials Chemistry Division, Chemistry Department, University of Helsinki, A.I. Virtasen Aukio 1, P.O. Box 55, FI- \\ 00014, Helsinki, Finland \\ ${ }^{\tau}$ Institute for Chemistry of Renewables, Department of Chemistry, University of Natural Resources and Life Sciences Vi- \\ enna, (BOKU), Muthgasse 18, A-1190 Wien, Austria
}

KEYWORDS. Mutarotation; C-glycoside; nanocellulose; cellobiose

\begin{abstract}
Herein, we demonstrate an effective approach towards functionalization of cellulose nanocrystal (CNC) reducing ends by means of a Knoevenagel condensation reaction with a reactive $\beta$-diketone (acetylacetone). The end-wise modification was elucidated by advanced NMR analysis, which was facilitated by dissolving the CNCs in ionic liquid electrolyte and by the concomitant assignment of a model compound derived from D-cellobiose. The diffusion-edited ${ }^{1} \mathrm{H}$ experiment afforded a simple method to identify the assigned model resonances in the reducing end modified CNCs. The condensations can be carried out in aqueous bicarbonate solutions, avoiding the use of hazardous solvents. Under these preliminary aqueous conditions, end-group conversion of up to $12.5 \%$ could be confirmed. These results demonstrate the potential of $\beta$-diketone chemistry and the Knoevenagel condensation for functionalizing cellulose reducing ends. Application of this liquid-state NMR method for confirming and quantifying reducing end conversion is also shown to be invaluable. Extension of this chemistry to other 1,3-dicarbonyl compounds and solvation conditions should allow for the topochemical and (axially) chirotopic installation of functional moieties to CNCs, paving the way to asymmetric cellulosebased nanomaterials with unique properties.
\end{abstract}

Cellulose nanocrystals (CNCs) are polymeric nanoscale building blocks with substantial potential for high-end material applications. They are isolated top-down from the plant cell wall, typically by sulfuric acid hydrolysis, resulting in charged, rod-shaped nanoparticles. The current popularity behind CNCs is based on their inimitable material characteristics, including long-range chirality inducing chiral nematic liquid crystal behavior $^{1-3}$, high tensile properties for composite reinforcement ${ }^{4-}$ ${ }^{6}$, and a unique water-response, allowing for formation of colloidal gels ${ }^{7}$ even before augmenting their surface chemistry. The materials spectrum of CNCs, however, can be widely expanded by tailored topochemical modifications. CNCs thereby serve as multifunctional templates bearing reactive surface hydroxyl groups and, effectively, aldehyde (hemiacetal) groups exclusively on one crystallite end owing to the inherent directionality of coalescent cellulose I chains. ${ }^{8}$ These so-called reducing end-groups (REs) would, thus, allow for an asymmetric and axially chirotopic installation of new, non-cellulosic functionality enabling fundamental changes in the CNC characteristics, e.g., dispersion behavior or colloidal self-assembly that might open up further application areas.

Published attempts that selectively targeted cellulose REs have used aldehyde-specific chemistry including (i) Pinnick oxidation, followed by amide formation ${ }^{9-13}$, (ii) reductive amination $^{9,14,15}$, and (iii) hydrazone formation ${ }^{16-19}$. Applied to CNCs, these protocols have, for instance, aimed at an asymmetric installation of marker molecules for end-group characterization ${ }^{20}$ or polymerization initiation sites ${ }^{10,11}$. All aldehyde-specific approaches, however, face one serious synthetic challenge: the availability of the REs' aldehyde reactive intermediary species is strongly limited by tautomeric equilibria favoring the stabilized hemiacetals. ${ }^{21,22}$ The free aldehyde form is, thus, only the transient open-chain intermediate of mutarotation, which involves proton-transfer in the transition between cyclic $\alpha$ - and $\beta$ anomers of reducing end-terminal anhydrous glucose units. ${ }^{23}$ Even though in water the velocity of mutarotation increases with temperature, the proportion of free aldehydes remains far below one percent (e.g. $0.02 \%$ aldehyde-D-glucose at $80{ }^{\circ} \mathrm{C}$ ), and only strongly acidic conditions have been shown to force the equilibrium towards the open-chain form ("reducing sugar"). ${ }^{21,24,25}$

Beside the synthetic aspects, end-wise modifications on CNCs confront us with a true analytical challenge considering the low abundance of introduced functionalities and given the low resolution or detection limits of common analytical methods, e.g., IR spectroscopy, elemental analysis, solid-state NMR. Therefore, most contributions published so far have relied on confirmation of end-wise functionalization using indirect methods, such as microscopy or measuring changes in physicochemical properties of the modified CNCs (e.g. dispersion behavior, 
thermal degradation, thermo-response ${ }^{9,10,14}$ ) rather than a profound analytical confirmation. These methods often leave behind the question of whether covalent modifications on the REs have taken place, or rather compound adsorption on the nanocrystal surface. Based on both synthetic and analytical challenges we, thus, aimed for: (i) alternative selective, aldehyde-specific reactions that activate and functionalize the anomeric center, and (ii) a highly sensitive analytical approach that unequivocally determines the end-wise modification.

We tackled the latter point by using accessible liquid-state NMR techniques, facilitated by dissolving the modified CNCs in the ionic liquid electrolyte tetrabutylphosphonium acetate ([P 4444$][\mathrm{OAc}]): \mathrm{DMSO}-d_{6}(1: 4 \mathrm{w} / \mathrm{w}) .{ }^{26}$ This powerful approach gives us the unique possibility to confirm the end-wise modification, assign the newly introduced signals at the REs, and to estimate their conversion. Additionally, the ketone adduct of D-cellobiose was used as a low-molecular-weight model compound, allowing for more profound spectral assignment to compare with the CNC spectra. For the synthetic part, we targeted the REs by a Knoevenagel-type condensation using reactive $\beta$-dicarbonyl compounds and acetylacetone (acac) as first simple representative for a group of potential reactants. Thus, we propose a novel synthetic concept for the modification of cellulose REs. Applied to mono- and disaccharides, reactions with acac have been shown to result in the formation of C-glycoside ketones with high yield and excellent stereoselectivity. ${ }^{27-29}$ Translated to more complex 1,3-dicarbonyl compounds, this reaction offers the potential to install arbitrary functionalities, in one step, to the REs of unprotected sugars (e.g. fatty $\beta$-diketones yielding $\mathrm{C}$-glycolipids ${ }^{30}$ ). Moreover, the condensations can be carried out as a one-pot reaction in aqueous alkaline (bicarbonate) media. The mechanism (Scheme 1) of Knoevenagel condensation thereby follows the initial condensation of carbanion and open-chain RE aldehyde, water elimination and cyclization to an intermediate Cglycoside, that finally undergoes retro-Claisen aldolization under basic conditions. ${ }^{31,32}$ Applied to $\mathrm{CNCs}$, it was particularly interesting for us to see whether a diketone can access the cellulose REs under heterogeneous Knoevenagel condi- tions, expecting a reduced accessibility of this highly crystalline substrate in dispersion compared to low-molecularweight sugars or polysaccharides in solution. Furthermore, we were aiming at investigating the exact impact of different obvious reaction conditions (incl. temperature, duration and bicarbonate concentration) on the accessibility and functionalization of CNC REs.

Before the reaction was translated to the more complex, heterogeneous cellulose system, D-cellobiose was functionalized in a water-based overnight reaction. The unprotected disaccharide was thereby converted quantitatively with acac (1.2 eq.) in the presence of sodium bicarbonate (1.5 eq.) at $90{ }^{\circ} \mathrm{C}$, and the corresponding $\mathrm{C}$-glycoside was obtained in good purity by a simple precipitation from methanol (see Supporting Information (SI Section S3b). The high conversion of cellobiose during Knoevenagel condensation can be very likely attributed to the activating effect of the bicarbonate, acting as both proton acceptor and donor in solution.

Scheme 1. Condensation of cellobiose with acac in aqueous bicarbonate and translation of the reaction to CNCs.

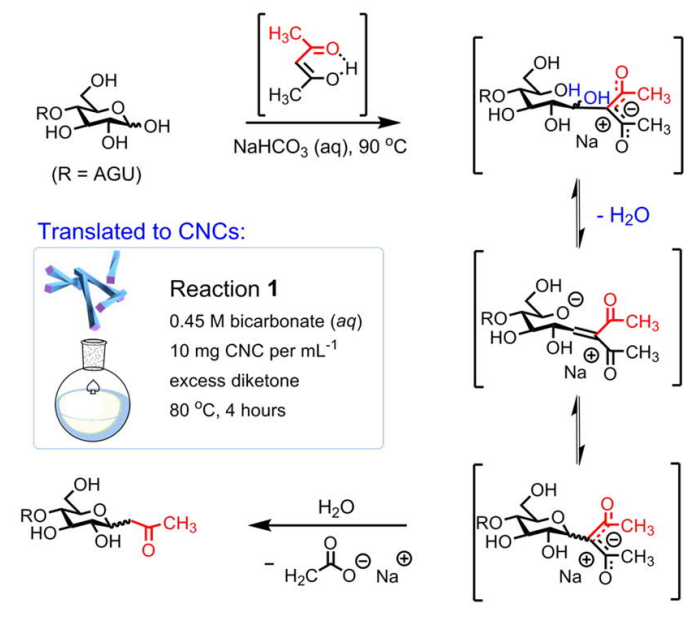




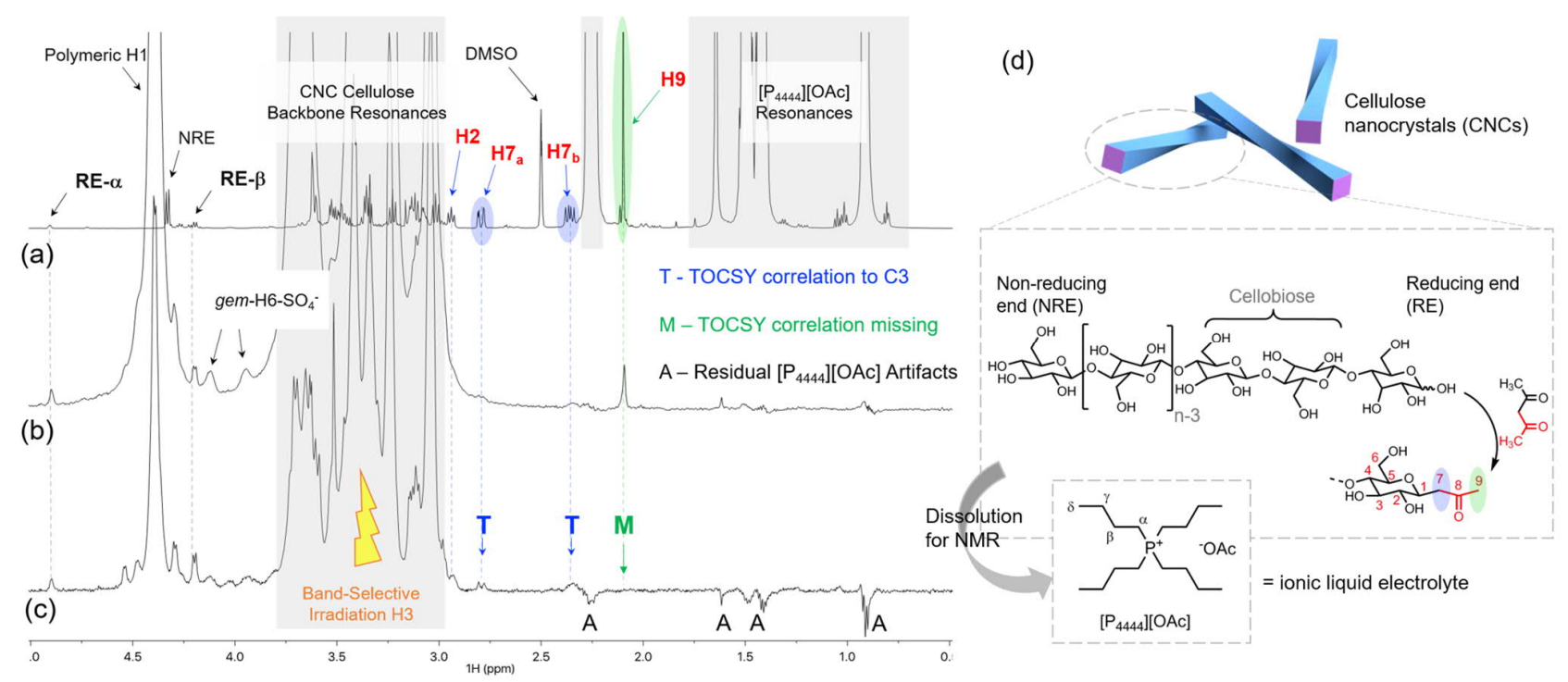

Figure 2. NMR spectra at $65^{\circ} \mathrm{C}$ in $\left[\mathrm{P}_{4444}\right][\mathrm{OAc}]: D M S O-d_{6}(1: 4 \mathrm{w} / \mathrm{w})$ : (a) Standard ${ }^{1} \mathrm{H}$ for cellobiose-ketone, (b) diffusion-edited ${ }^{1} \mathrm{H}$ (with fast-diffusing species filtered out), (c) band-selective TOCSY (selective irradiation of the H3-region) for CNC-ketone 1, showing correlations to $\mathrm{H} 7$ only; and (d) schematic illustration of $\mathrm{CNC}$ cellulose molecular structure before and after RE modification, and molecular structure of ionic liquid electrolyte used for NMR analysis.

The monobasic bicarbonate, thus, promotes the deprotonation of acac, but also the ring-opening (mutarotation) of the RE, which can be both acid and base-catalyzed. ${ }^{33,34}$ Standard ${ }^{1} \mathrm{H}$ NMR analysis of the product in $\mathrm{D}_{2} \mathrm{O}$ (Figure $\mathrm{S} 4$ ) already revealed the introduction of a strong acetyl signal $(2.28 \mathrm{ppm}-$ $\mathrm{CH}_{3}$ ) together with an almost complete disappearance of the REs' $^{\prime} \beta$-hemiacetal signal (4.66 ppm) as a consequence of the ketone functionalization. For detailed structure elucidation, multiplicity-edited HSQC and HSQC-TOCSY (shortrange/15 ms mixing time and long-range/120 ms mixing time) were performed at $65^{\circ} \mathrm{C}$ on the model compound, dissolved in $\left[\mathrm{P}_{4444}\right][\mathrm{OAc}]: \mathrm{DMSO}-d_{6}(1: 4 \mathrm{w} / \mathrm{w}$, see SI Section S4). As shown in Figure 1(a), all cellobiose ring signals of the RE and non-reducing end (NRE) are clearly resolved. In this solvent system, the methyl ${ }^{1} \mathrm{H}$ (9) peak shifted up-field to $2.09 \mathrm{ppm}$. Moreover, two additional signals at $2.36 \mathrm{ppm}$ and $2.79 \mathrm{ppm}$ in the ${ }^{1} \mathrm{H}$ spectral range, and $46.23 \mathrm{ppm}$ in ${ }^{13} \mathrm{C}$ spectral range, can be assigned to the newly introduced geminal-methylene group of the ketone functionality. TOCSY correlations (longrange) can be used to trace the full spin-systems for the separate 'sugar units' (Figure 1(b)). A weak correlation can further be observed linking C7 and C9 over the C8 carbonyl. Additionally, a HMBC experiment (Figure S6) was used to show a correlation between the $\mathrm{C} 8$ carbonyl and the adjacent $\mathrm{CH}$ positions (C7 \& C9).

DFT (density functional theory) was used to calculate ${ }^{1} \mathrm{H}$ and ${ }^{13} \mathrm{C}$ chemical shifts for the expected $\beta$-isomer and unexpected $\alpha$-isomer, using glucose as a model sugar (SI Section S4d). The ${ }^{1} \mathrm{H}$ and ${ }^{13} \mathrm{C}$ NMR chemical shifts were calculated using the ORCA 4.1.2 package $^{35}$ with the M06-L functional and pc-Sseg-3 basis set and implicit conductor-like polarizable continuum model (CPCM) solvation (DMSO). Correlation plots for the isomers are shown in Figure S10, where it is clearly shown that the $\alpha$-isomer displays a large downfield shift for the calculated $\mathrm{H} 7{ }^{1} \mathrm{H}$ resonances. The calculated $\mathrm{H} 7$ resonances for the $\beta$-isomer are largely consistent with the corresponding experimental values, proving that the cellobiose ketone is the $\beta$-isomer, which is also consistent with previous reports. $^{31}$

After successful assignment of the model compound, we translated the reaction to CNCs. Rod-shaped nanocrystals (average size: $125.5 \times 6.7 \mathrm{~nm}$ ) were obtained by hydrolysis in 64 wt $\%$ sulfuric acid using a well-established procedure, ${ }^{36,37}$ starting from cotton linter filter paper (preparation and characterization in SI Section S2). To ensure a good redispersibility and high colloidal stability in water, after freeze-drying, the $\mathrm{CNCs}$ were used in their sodium salt form $\left(-\mathrm{OSO}_{3} \mathrm{Na}\right.$, $0.30 \mathrm{mmol} / \mathrm{g}$; zeta potential $(\zeta)-39.4 \mathrm{mV}$ at $\mathrm{pH} 5.18$ ). For NMR analysis, 5 wt $\%$ of the freeze-dried CNCs were dissolved in $\left[\mathrm{P}_{4444}\right][\mathrm{OAc}]: \mathrm{DMSO}-d_{6}(1: 4 \mathrm{w} / \mathrm{w}$, see SI Section $\mathrm{S} 4 \mathrm{~b})$ at RT. As described previously ${ }^{26}$, a sample temperature of $65{ }^{\circ} \mathrm{C}$ was the optimum for giving good signal-to-noise and resolution, without decomposing the sample. Additionally, diffusion-editing gave the possibility to distinguish between cellulose-derived (slow-diffusing) and ionic liquid (fast-diffusing) resonances. ${ }^{26}$ The latter would otherwise strongly overlap with the spectral region of the newly introduced ketone species (Figure S5), whereas, in the 1D diffusion-edited spectra only artifacts of ionic liquid peaks remain. The sensitivity of this powerful NMR technique can be demonstrated by the fact that we are able to observe signals corresponding to both the anomeric REs, NREs, remaining sulfate half-esters on C6 and the introduced ketone groups, which are not resolvable using conventional solid-state NMR methods.

A partial conversion of CNC REs was already reached in a 4-hour reaction in $0.45 \mathrm{M}$ bicarbonate $(\mathrm{pH} 8.5)$ solution at $80^{\circ} \mathrm{C}$ and with an excess of acac (Table 2). Site-specific conversion was thereby confirmed by correlating ${ }^{1} \mathrm{H}$ NMR signals of the cellobiose ketone and CNC-ketone 1 (Figure 2). Addi- 
tionally, a band-selective TOCSY experiment (selective irradiation of the $\mathrm{H} 3$ region) gives clear TOCSY correlations to the methylene protons of the ketone functionality, whereas the H3-H9 TOCSY correlations are missing (Figure 2(c)), as would typically be expected for coupling over 3 bonds.

The relative percentage degrees of RE conversion to ketone $\left(\mathrm{DC}_{\%}\right)$ were determined by deconvolution of the diffusion-edited ${ }^{1} \mathrm{H}$ spectra. Deconvolution of the main polymeric $\mathrm{H} 1$ peak vs the ketone H9 peak in the diffusion-edited spectra (SI Section S4f, Figure S16 \& S17) yielded the most accurate relative values for $\mathrm{DC}_{\%}$, due to the clear separation of the ketone $\mathrm{H} 9$ signal after editing out the overlapping low molecular weight species. However, the diffusion-edited experiment is not quantitative so a correlation was drawn between the $\mathrm{DC}_{\%}$ values obtained from the deconvolution of the quantitative ${ }^{1} \mathrm{H}$ spectra and the diffusion-edited ${ }^{1} \mathrm{H}$ spectra. This gave a linear correlation of sufficient accuracy (Figure S17) to determine the correct magnitude of the $\mathrm{DC}$ \% values, based on the quantitative ${ }^{1} \mathrm{H}$ deconvolutions. The number-average degree of polymerization $\left(\mathrm{DP}_{\mathrm{n}}\right)$ for pristine CNCs was also obtained from deconvolution of the polymeric $\mathrm{H} 1$ vs NRE, $\mathrm{RE}_{\alpha}$ and $\mathrm{RE}_{\beta}$ peaks. The $\mathrm{DP}_{\mathrm{n}}$ value for the pristine $\mathrm{CNCs}$, based on the NMR deconvolutions (SI S4e \& Figure S15), was then used for $\mathrm{DC}_{\%}$ determination (according to equation $\mathrm{S} 1$ ). All ${ }^{1} \mathrm{H}$ NMR spectra and full DC\% calculation details are in the SI (Sections S4e and S4f). In order to further validate the NMRbased $\mathrm{DP}_{\mathrm{n}}, \mathrm{GPC}$ was used to measure the molar mass distribution (MMD) of the pristine CNCs using either a conventional pullulan calibration method or multi-angle light-scattering (MALS) (see SI Section S2b). Additionally, the REs of the unmodified CNCs were quantified using a fluorescent labelling with carbazole-9-carboxylic acid [2-(2-aminooxyethoxy)ethoxy]amide (CCOA) (see SI Section S2b) ${ }^{38}$ A summary of the obtained data using the different techniques is shown in Table 1.

Table 1. Number average molar masses $\left(\mathrm{M}_{\mathrm{n}}\right)$, degrees of polymerization $\left(\mathrm{DP}_{\mathrm{n}}\right)$, and REs obtained from GPC, NMR, or CCOA labelling.

\begin{tabular}{cccc} 
Technique & $\mathrm{M}_{\mathrm{n}}[\mathrm{kDa}]$ & $\mathrm{DP}$ & $\mathrm{REs}\left[\mu \mathrm{mol} \mathrm{g}^{-1}\right]$ \\
GPC - Pullulan & 16.3 & 101 & 61.3 \\
GPC - MALS & 21.4 & 132 & 46.7 \\
NMR & 10.5 & 65 & 95.1 \\
CCOA & 11.9 & 74 & 84.0 \\
\hline
\end{tabular}

As a consequence of the acid treatment, the CNCs exhibited a multimodal MMD (Figure S2 and S3). The error for both MALS and pullulan calibration can be considered as relatively high owing to the overall low MM of the CNCs reducing the signal-to-noise of MALS signals, and a non-ideal relationship between MM and retention time throughout the GPC separation. Also, the CCOA labelling showed a relatively high error (see Table S1) and indicated the presence of extra keto groups in the sample implying a random oxidation along the cellulose chains. Nevertheless, the values for the $\mathrm{DP}_{\mathrm{n}}$ and the REs obtained from the different analysis techniques are in a relatively good agreement, with the NMR and CCOA methods showing quite similar values. The results further emphasize the merit of the NMR method when dealing with relatively low DP samples, such as CNCs. MM determinations for cellulose samples with GPC (low and high MM) are still challenging and the data evaluation is strongly affected by the operator (e.g. baseline alignments, peak selection).

DC\% for CNC-ketone 1 was estimated to be 7\%, which is very low compared to the almost quantitative conversion of cellobiose. We, therefore, aimed at further increasing the endgroup conversion by (i) increasing reaction time and temperature (CNC-ketones 2-4), considering mutarotation as the limiting factor, (ii) repeated reagent addition (CNC-ketone 4), and by (iii) reducing $\mathrm{CNC}$ aggregation (potentially salt or CNC concentration-induced, CNC-ketone 5), or by using never-dried CNCs (CNC-ketone 6). The exact parameter sets and resulting $\mathrm{DC}_{\%}$ are listed in Table 2.

Table 2. Reaction conditions for Knoevenagel-type condensations with acetylacetone on CNC REs

\begin{tabular}{cccccc}
$\begin{array}{c}\mathrm{CNC}- \\
\text { ketone }\end{array}$ & $\begin{array}{c}\mu \mathrm{mol} \\
\mathrm{acac}^{a}\end{array}$ & $\begin{array}{c}\mathrm{NaHCO}_{3} \\
{\left[\mathrm{~mol} \mathrm{~L}^{-1}\right]}\end{array}$ & $\begin{array}{c}\mathrm{CNC} \\
{\left[\mathrm{mg} \mathrm{mL}^{-1}\right]}\end{array}$ & $\begin{array}{c}\mathrm{T}\left[{ }^{\circ} \mathrm{C}\right], \\
\mathrm{t}[\mathrm{h}]\end{array}$ & $\mathrm{DC}_{\%}^{d}$ \\
1 & 800 & 0.45 & 10 & 80,4 & 6.9 \\
2 & 800 & 0.45 & 10 & 80,24 & 10.6 \\
3 & 800 & 0.45 & 10 & 90,24 & 2.8 \\
4 & $2 \mathrm{x}$ & 0.45 & 10 & 80,48 & 12.5 \\
& $800^{b}$ & & & & \\
5 & 800 & 0.10 & 5 & 80,24 & 3.3 \\
6 & 800 & 0.45 & $\mathrm{nd}^{c}$ & 80,24 & 2.4 \\
\hline
\end{tabular}

${ }^{a}$ acac - acetylacetone, per $200 \mathrm{mg} \mathrm{CNC} ;{ }^{b}$ second reagent addition after $24 \mathrm{~h} ;{ }^{c}$ use of never-dried $-\mathrm{OSO}_{3} \mathrm{H}-\mathrm{CNC}$ suspension $\left(5.5 \mathrm{mg} \mathrm{mL}{ }^{-1}\right.$, see SI Section S3b); ${ }^{d} \mathrm{DC}=$ degree of conversion estimated from diffusion-edited ${ }^{1} \mathrm{H}$ NMR spectra (calculation SI Section S4f)

An increase of the reaction temperature by $10{ }^{\circ} \mathrm{C}$ reduced the RE conversion significantly by more than $50 \%$ (CNCketone 3), irrespective of the expected enhancement of the mutarotation velocity and reaction rate. In contrast, the $\mathrm{DC} \%$ increased with the reaction time and when fresh reagents were added after the first half of the reaction (CNC-ketone 4). The increase in end-group conversion as a function of time can be observed from the diffusion-edited ${ }^{1} \mathrm{H}$ spectra, with an increase in intensity for the ketone H9 signal (Figure 3).

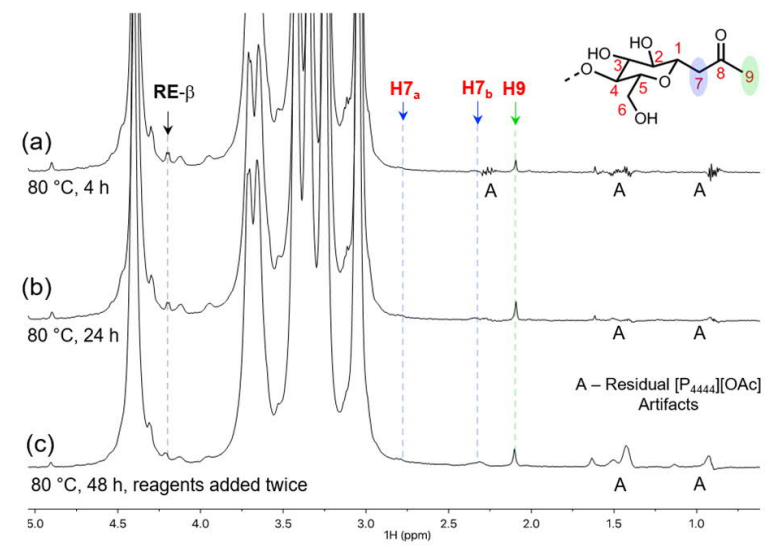

Figure 3. Condensations with acetylacetone - diffusion-edited ${ }^{1} \mathrm{H}$ spectra at $65{ }^{\circ} \mathrm{C}$ in $\left[\mathrm{P}_{4444}\right][\mathrm{OAc}]: \mathrm{DMSO}-d_{6}(1: 4 \mathrm{w} / \mathrm{w})$ for (a) CNC-ketone 1, (b) CNC-ketone 2 and (c) CNC-ketone 4.

Nevertheless, the overall conversion remained fairly low in this reaction system. Beside the mutarotation kinetics, ionic 
strength-induced aggregation was considered as a main factor limiting the accessibility of CNC REs. It has been shown that the presence of monovalent cations destabilizes CNC suspensions, which dramatically increases with the salinity (e.g. for $\mathrm{NaCl}$ between 2 to $200 \mathrm{mmol} \mathrm{L}^{-1}$ ) forming side-by-side lateral and branched $\mathrm{CNC}$ aggregates. ${ }^{39,40}$ Conversely, even though $\mathrm{CNC}$ aggregation might have been reduced for CNC-ketone $\mathbf{5}$ (lower bicarbonate concentration), the increased dilution factor of the system obviously complicated the reaction. Moreover, bicarbonate was still introduced in large excess to both the diketone (acac:bicarbonate $=1: 5 \mathrm{~mol} / \mathrm{mol}$ ) and the REs. Therefore, a hampered reagent and end-group "activation" is rather unlikely as a reason for the diminished conversion in reaction 5. Freeze-drying before the chemical modification was considered as another factor causing (partially) irreversible CNC aggregation and, thus, a reduced RE accessibility. Therefore, we expected a significantly higher functionalization for reactions starting from never-dried nanocrystals (CNC-ketone 6). These CNCs were freshly hydrolyzed and purified, and used in their original protonated form $\left(-\mathrm{OSO}_{3} \mathrm{H}\right)$. This also excludes a potential salt-induced aggregation during the $\mathrm{pH}$ adjustments throughout the CNC preparation (see SI Section S2a). Nevertheless, compared to reaction 2, the DC was noticeably reduced, which might be again a consequence of the high dilution factor ( $5.5 \mathrm{mg} \mathrm{CNC}$ per $\mathrm{mL}$ ). Overall, the highest $\mathrm{DC} \%$ of $12.5 \%$ was reached in a 48 -hours reaction, with a second diketone addition after 24 hours. This is a very common approach to increase the reagent concentration gradient forcing the reaction equilibrium toward the product. ${ }^{14}$ Why the conversion was then restricted to less than $20 \%$ likely reflects the complex interplay between RE mutarotation kinetics and the applied reaction conditions, whereby the reaction time might have been the limiting factor here. Compared to the applied Knoevenagel conditions, the CCOA labelling, where we used an oxime reagent for end-group analysis, presumably gave a full conversion (see Table 1 ) within 7 days at a pH of 4.5 (see SI Section S2b). That a quantitative conversion of reducing sugars can also be realized under mildly basic conditions, has been demonstrated by our model compound cellobiose. However, even in the case of the disaccharide, the condensation reaction proceeded relatively slow (>12 h). Combined with the expectedly restricted accessibility of the highly crystalline $\mathrm{CNCs}$, significantly longer reaction times might be the key towards a quantitative conversion of the cellulose REs. Moreover, it has been shown that CNCs, even when freshly hydrolyzed, often exist in complexes of laterally assembled elementary crystallites, rather than single rods ${ }^{41}$, which might reduce the accessibility of the individual CNCs' REs further. Whether these issues affect other CNC RE modification strategies is not clear, due to the lack of suitable direct analytics, up until now. Benchmarking of these systems using liquid-state NMR now seems to be a logical progression of this work.

In conclusion, we can state that Knoevenagel condensations using reactive 1,3-dicarbonyl compounds are a selective way to target the REs of CNCs. Combined with the advanced liquid-state NMR techniques used in this study, one is able to establish the topochemical CNC modification using a direct analytical method. However, in order to increase the REs' conversion, further studies on the condensation mechanism would be important. These should, particularly, explore the long reaction durations and, additionally, the possibility of using alternative solvent systems to improve dispersion (reduce aggregation). Moreover, we envisage that this chemistry may be translated to more complex 1,3-dicarbonyl compounds offering the potential to install a library of different molecular building blocks in one step, topochemically and chirotopically, to CNC REs.

\section{ASSOCIATED CONTENT}

\section{Supporting Information}

The Supporting Information is available free of charge on the ACS Publications website.

Materials; CNC preparation and characterization, synthesis cellobiose and CNC ketones; NMR characterization: sample preparation, measurement protocols, DFT calculations, degree of conversion $(\mathrm{PDF})$

\section{AUTHOR INFORMATION}

\section{Corresponding Author \\ * alistair.king@helsinki.fi, mauri.kostiainen@ aalto.fi, eero.kontturi@aalto.fi}

\section{Author Contributions}

The manuscript was written through contributions of all authors. All authors have given approval to the final version of the manuscript.

\section{Funding Sources}

$\mathrm{KH}$ and EK gratefully acknowledge the FinnCERES program enabled by the Academy of Finland, Aalto University and VTT. AK and TK would like to acknowledge the Academy of Finland for funding under the Project "WTF-Click-Nano" (311255).

\section{ACKNOWLEDGMENT}

The authors gratefully acknowledge Mrs. Rita Hatakka for carrying out SEC measurements, and the CSC - IT Center for Science, Finland, for computational resources. This work made use of Aalto University's Bioeconomy Facilities and the FinnCERES ecosystem.

\begin{abstract}
ABBREVIATIONS
Acac, acetylacetone; CCOA, carbazole-9-carboxylic acid [2-(2aminooxyethoxy)ethoxy]amide; CNCs, cellulose nanocrystals; $\mathrm{DC} \%$, degree of (end-group) conversion; DFT, density functional theory; DP, degree of polymerization; HMBC, heteronuclear multiple bond correlation; HSQC, heteronuclear single quantum coherence; nd, never-dried; MM, molar mass; MMD, molar mass distribution; NRE, non-reducing end-group; RE, reducing endgroup; RT, room temperature; TOCSY, total correlation spectroscopy
\end{abstract}

\section{REFERENCES}

(1) Shopsowitz, K. E.; Qi, H.; Hamad, W. Y.; MacLachlan, M. J. Free-Standing Mesoporous Silica Films with Tunable Chiral Nematic Structures. Nature 2010, 468 (7322), 422-425. https://doi.org/10.1038/nature09540.

(2) Giese, M.; Blusch, L. K.; Khan, M. K.; MacLachlan, M. J. Functional Materials from Cellulose-Derived LiquidCrystal Templates. Angew. Chem. Int. Ed. 2015, 54 (10), 2888-2910. https://doi.org/10.1002/anie.201407141.

(3) Li, Y.; Jun-Yan Suen, J.; Prince, E.; Larin, E. M.; Klinkova, A.; Thérien-Aubin, H.; Zhu, S.; Yang, B.; 

teric Liquid Crystal in Spherical Confinement. Nat. Commun. 2016, $\quad 7, \quad 12520$. https://doi.org/10.1038/ncomms12520.

Fortunati, E.; Armentano, I.; Zhou, Q.; Puglia, D.; Terenzi, A.; Berglund, L. A.; Kenny, J. M. Microstructure and Nonisothermal Cold Crystallization of PLA Composites Based on Silver Nanoparticles and Nanocrystalline Cellulose. Polym. Degrad. Stab. 2012, 97 (10), 2027-2036. https://doi.org/10.1016/j.polymdegradstab.2012.03.027.

(5) McKee, J. R.; Hietala, S.; Seitsonen, J.; Laine, J.; Kontturi, E.; Ikkala, O. Thermoresponsive Nanocellulose Hydrogels with Tunable Mechanical Properties. ACS Macro Lett. 2014, 3 (3), 266-270. https://doi.org/10.1021/mz400596g.

(6) Sapkota, J.; Natterodt, J. C.; Shirole, A.; Foster, E. J.; Weder, C. Fabrication and Properties of Polyethylene/Cellulose Nanocrystal Composites. Macromol. Mater. Eng. 2017, $\quad 302 \quad$ (1), 1600300. https://doi.org/10.1002/mame.201600300.

(7) Lewis, L.; Hatzikiriakos, S. G.; Hamad, W. Y.; MacLachlan, M. J. Freeze-Thaw Gelation of Cellulose Nanocrystals. ACS Macro Lett. 2019, 486-491. https://doi.org/10.1021/acsmacrolett.9b00140.

(8) Hieta, K.; Kuga, S.; Usuda, M. Electron Staining of Reducing Ends Evidences a Parallel-Chain Structure in Valonia Cellulose. Biopolym. Orig. Res. Biomol. 1984, 23 (10), 1807-1810.

(9) Arcot, L. R.; Lundahl, M.; Rojas, O. J.; Laine, J. Asymmetric Cellulose Nanocrystals: Thiolation of Reducing End Groups via NHS-EDC Coupling. Cellulose 2014, 21 (6), 4209-4218. https://doi.org/10.1007/s10570-0140426-9.

(10) Zoppe, J. O.; Dupire, A. V. M.; Lachat, T. G. G.; Lemal, P.; Rodriguez-Lorenzo, L.; Petri-Fink, A.; Weder, C.; Klok, H.-A. Cellulose Nanocrystals with Tethered Polymer Chains: Chemically Patchy versus Uniform Decoration. ACS Macro Lett. 2017, 6 (9), 892-897. https://doi.org/10.1021/acsmacrolett.7b00383.

(11) Risteen, B.; Delepierre, G.; Srinivasarao, M.; Weder, C.; Russo, P.; Reichmanis, E.; Zoppe, J. Thermally Switchable Liquid Crystals Based on Cellulose Nanocrystals with Patchy Polymer Grafts. Small 2018, 14 (46), 1802060. https://doi.org/10.1002/smll.201802060.

(12) Villares, A.; Moreau, C.; Cathala, B. Star-like Supramolecular Complexes of Reducing-End-Functionalized Cellulose Nanocrystals. ACS Omega 2018, 3 (11), 1620316211. https://doi.org/10.1021/acsomega.8b02559.

(13) Lin, F.; Cousin, F.; Putaux, J.-L.; Jean, B. TemperatureControlled Star-Shaped Cellulose Nanocrystal Assemblies Resulting from Asymmetric Polymer Grafting. ACS Macro Lett. 2019, 8 (4), 345-351. https://doi.org/10.1021/acsmacrolett.8b01005.

(14) Lokanathan, A. R.; Nykänen, A.; Seitsonen, J.; Johansson, L.-S.; Campbell, J.; Rojas, O. J.; Ikkala, O.; Laine, J. CiliaMimetic Hairy Surfaces Based on End-Immobilized Nanocellulose Colloidal Rods. Biomacromolecules 2013, 14 (8), 2807-2813. https://doi.org/10.1021/bm400633r.

(29) Carpenter, C. A.; Kenar, J. A.; Price, N. P. Preparation of Saturated and Unsaturated Fatty Acid Hydrazides and Long Chain C-Glycoside Ketohydrazones. Green Chem. 2010, 12 (11), 2012-2018.

Yagi, S.; Kasuya, N.; Fukuda, K. Synthesis and Characterization of Cellulose- $b$-Polystyrene. Polym. J. 2010, 42 (4), 342-348. https://doi.org/10.1038/pj.2009.342. ducing Ends of Bacterial Cellulose. Carbohydr. Res. 1988, $180(2), 345-350$

Koyama, M.; Helbert, W.; Imai, T.; Sugiyama, J.; Henrissat, B. Parallel-up Structure Evidences the Molecular Directionality during Biosynthesis of Bacterial Cellulose. Proc. Natl. Acad. Sci. 1997, 94 (17), 9091-9095.

Sipahi-Sağlam, E.; Gelbrich, M.; Gruber, E. Topochemically Modified Cellulose. Cellulose 2003, 10 (3), 237-250. https://doi.org/10.1023/A:1025151701985.

Kim, N.-H.; Imai, T.; Wada, M.; Sugiyama, J. Molecular Directionality in Cellulose Polymorphs. Biomacromole$\begin{array}{lllll}\text { cules 2006, } 7 \text { (1), 274-280. } & \end{array}$ https://doi.org/10.1021/bm0506391.

Huang, J.-L.; Li, C.-J.; Gray, D. G. Cellulose Nanocrystals Incorporating Fluorescent Methylcoumarin Groups. ACS Sustain. Chem. Eng. 2013, 1 (9), 1160-1164. https://doi.org/10.1021/sc400074e.

Lowry, T. M.; Smith, G. F. The Mutarotation of Galactose. J. Phys. Chem. 1929, 33 (1), 9-21.

Wolfrom, M. L. The Acetate of the Free Aldehyde Form of Glucose. J. Am. Chem. Soc. 1929, 51 (7), 2188-2193.

Ballash, N. M.; Robertson, E. B. The Mutarotation of Glucose in Dimethylsulfoxide and Water Mixtures. Can. J. Chem. 1973, 51 (4), 556-564. https://doi.org/10.1139/v73085 .

Maple, S. R.; Allerhand, A. Detailed Tautomeric Equilibrium of Aqueous D-Glucose. Observation of Six Tautomers by Ultrahigh Resolution Carbon-13 NMR. $J$. Am Chem. Soc. 1987, 109 (10), 3168-3169.

Pacsu, E.; Hiller Jr, L. A. Open-Chain Sugars. I. Absorption in Ultraviolet of D-Glucose and L-Arabinose in Acid Solution. J. Am. Chem. Soc. 1948, 70 (2), 523-526.

King, A. W. T.; Mäkelä, V.; Kedzior, S. A.; Laaksonen, T.; Partl, G. J.; Heikkinen, S.; Koskela, H.; Heikkinen, H. A.; Holding, A. J.; Cranston, E. D.; et al. Liquid-State NMR Analysis of Nanocelluloses. Biomacromolecules 2018. https://doi.org/10.1021/acs.biomac.8b00295. One-Step, Synthesis of $\beta$-C-Glycosidic Ketones in Aqueous Media. Chem. Commun. 2000, $O$ (20), 2049-2050. https://doi.org/10.1039/B006642G.

Riemann, I.; Fessner, W.-D.; Papadopoulos, M. A.; Knorst, M. C-Glycosides by Aqueous Condensation of $\beta$ Dicarbonyl Compounds with Unprotected Sugars. Aust. J. Chem. 2002, 55 (2), 147-154. https://doi.org/10.1071/ch02012.

Hersant, Y.; Abou-Jneid, R.; Canac, Y.; Lubineau, A.; 
Philippe, M.; Semeria, D.; Radisson, X.; Scherrmann, M.C. One-Step Synthesis of $\beta$-C-Glycolipid Derivatives from Unprotected Sugars. Carbohydr. Res. 2004, 339 (3), 741745. https://doi.org/10.1016/j.carres.2003.11.007.

Rodrigues, F.; Canac, Y.; Lubineau, A. A Convenient, One-Step, Synthesis of $\beta$-C-Glycosidic Ketones in Aqueous Media. Chem. Commun. 2000, O (20), 2049-2050. https://doi.org/10.1039/B006642G.

(32) Scherrmann, M.-C. Knoevenagel Reaction of Unprotected Sugars. In Carbohydrates in Sustainable Development II; Rauter, A. P., Vogel, P., Queneau, Y., Eds.; Topics in Current Chemistry; Springer Berlin Heidelberg: Berlin, Heidelberg, $2010 ; \quad$ pp $\quad 1-18$. https://doi.org/10.1007/128_2010_49.

(33) Murdock, M.; Holman, R. W.; Slade, T.; Clark, S. L. D.; Rodnick, K. J. An Introductory Organic Chemistry Review Homework Exercise: Deriving Potential Mechanisms for Glucose Ring Opening in Mutarotation. J. Chem. Educ. 2014, $\quad 91 \quad$ (12), 2146-2147. https://doi.org/10.1021/ed500307d.

(34) Rivlin, M.; Navon, G. 3-O-Methyl-d-Glucose Mutarotation and Proton Exchange Rates Assessed by 13C, 1H NMR and by Chemical Exchange Saturation Transfer and Spin Lock Measurements. J. Biomol. NMR 2018, 72 (1), 93-103. https://doi.org/10.1007/s10858-018-0209-y.

(35) Neese, F. The ORCA Program System. Wiley Interdiscip. Rev. Comput. Mol. Sci. 2012, 2 (1), 73-78. https://doi.org/10.1002/wcms.81.

(36) Edgar, C. D.; Gray, D. G. Smooth Model Cellulose I Surfaces from Nanocrystal Suspensions. Cellulose 2003, 10 (4), 299-306. https://doi.org/10.1023/A:1027333928715.
(37) Salminen, R.; Baccile, N.; Reza, M.; Kontturi, E. SurfaceInduced Frustration in Solid State Polymorphic Transition of Native Cellulose Nanocrystals. Biomacromolecules 2017, 18 (6), 1975-1982.

(38) Röhrling, J.; Potthast, A.; Rosenau, T.; Lange, T.; Ebner, G.; Sixta, H.; Kosma, P. A Novel Method for the Determination of Carbonyl Groups in Cellulosics by Fluorescence Labeling. 1. Method Development. Biomacromolecules 2002, 3 (5), 959-968. https://doi.org/10.1021/bm020029q.

(39) Cherhal, F.; Cousin, F.; Capron, I. Influence of Charge Density and Ionic Strength on the Aggregation Process of Cellulose Nanocrystals in Aqueous Suspension, as Revealed by Small-Angle Neutron Scattering. Langmuir 2015, $31 \quad$ (20), 5596-5602. https://doi.org/10.1021/acs.langmuir.5b00851.

(40) Phan-Xuan, T.; Thuresson, A.; Skepö, M.; Labrador, A.; Bordes, R.; Matic, A. Aggregation Behavior of Aqueous Cellulose Nanocrystals: The Effect of Inorganic Salts. Cellulose 2016, $23 \quad$ (6), 3653-3663. https://doi.org/10.1007/s10570-016-1080-1.

(41) Elazzouzi-Hafraoui, S.; Nishiyama, Y.; Putaux, J.-L.; Heux, L.; Dubreuil, F.; Rochas, C. The Shape and Size Distribution of Crystalline Nanoparticles Prepared by Acid Hydrolysis of Native Cellulose. Biomacromolecules 2008, 9 (1), 57-65. https://doi.org/10.1021/bm700769p. 
SYNOPSIS TOC

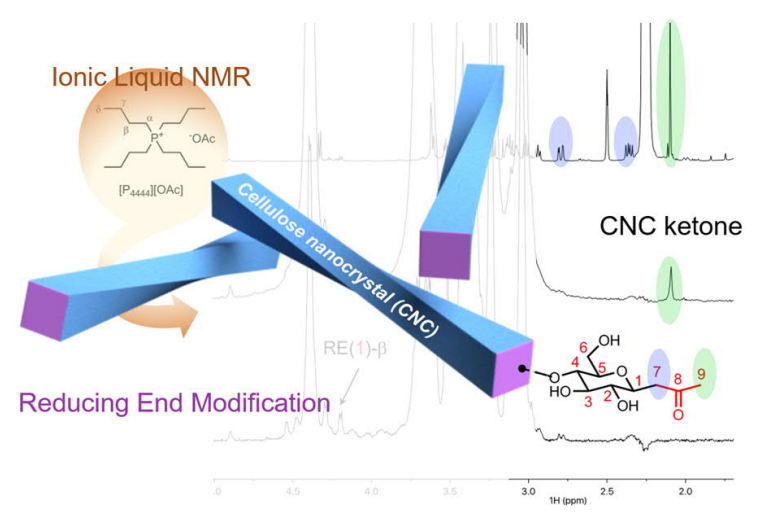

19

20

21

22

23

24

25

26

27 